\title{
Electromechanical Catastrophe
}

\section{Citation}

Lu, Tongqing, Sibo Cheng, Tiefeng Li, Tiejun Wang, and Zhigang Suo. 2016. Electromechanical Catastrophe. International Journal of Applied Mechanics 08, no. 07: 1640005. doi:10.1142/ s1758825116400056.

\section{Published Version}

$10.1142 / s 1758825116400056$

\section{Permanent link}

http://nrs.harvard.edu/urn-3:HUL.InstRepos:29995325

\section{Terms of Use}

This article was downloaded from Harvard University's DASH repository, and is made available under the terms and conditions applicable to Open Access Policy Articles, as set forth at http:// nrs.harvard.edu/urn-3:HUL.InstRepos:dash.current.terms-of-use\#OAP

\section{Share Your Story}

The Harvard community has made this article openly available.

Please share how this access benefits you. Submit a story.

Accessibility 


\title{
Electromechanical Catastrophe
}

\author{
Tongqing Lu
}

State Key Lab for Strength and Vibration of Mechanical Structures, Shaanxi Engineering Laboratory for Vibration Control of Aerospace Structures, Department of Engineering Mechanics, Xïan Jiaotong University,

Xi'an 710049, China

tongqinglu@mail.xjtu.edu.cn

Sibo Cheng

State Key Lab for Strength and Vibration of Mechanical Structures, International Center for Applied Mechanics, Xi’an Jiaotong University,

Xi'an 710049, China

lightl@stu.xjtu.edu.cn

Tiefeng Li

Key Laboratory of Soft Machines and Smart Devices of Zhejiang Province, Department of Engineering Mechanics, Zhejiang University, Hangzhou 310027, China litiefeng@zju.edu.cn

Tiejun Wang*

State Key Lab for Strength and Vibration of Mechanical Structures, Department of Engineering Mechanics, School of Aerospace Engineering, Xi'an Jiaotong University, Xi'an 710049, China

wangtj@mail.xjtu.edu.cn

Zhigang Suo*

School of Engineering and Applied Sciences, Kavli Institute of Bionano Science and Technology, Harvard University, Cambridge, Massachusetts o2138, USA International Center for Applied Mechanics, Xi'an Jiaotong University, Xi'an 710049, China suo@seas.harvard.edu 


\begin{abstract}
A transducer is a system that couples two loads. For example, an electromechanical transducer couples a mechanical force and an electrical voltage. A two-load, nonlinear system can exhibit rich behavior of bifurcation, which can be displayed in a three-dimensional space, with the horizontal plane representing the two loads, and the vertical axis representing the state of the system. In this three-dimensional space, a state of equilibrium at fixed loads corresponds to a point on a surface. The surface is smooth, but its projection to the load plane results in singularities of two types: fold and cusp. Here we identify the fold and cusp for a dielectric elastomer transducer by a combination of experiment and calculation. We conduct two kinds of experiment: electrical actuation under a constant force and mechanical pulling under a constant voltage. The theory and the experiment agree well. The fold and cusp are essential in the design of loading paths to avoid or harness the bifurcation.
\end{abstract}

Keywords: Dielectric elastomer; Bifurcation; Electromechanical instability; Catastrophe 


\section{Introduction}

When a time-independent, nonlinear system is subject to a single load, at a given value of the load, the system may reach no state of equilibrium, one state of equilibrium, or multiple states of equilibrium. As the load changes continuously, the state of the system usually changes continuously, but not always. At particular values of the load, the number of state of equilibrium may change, a phenomenon called bifurcation. Familiar examples include the buckling of a rod under compression, and the necking of a rod under tension [Landau and Lifshitz, 1986; Hill, 1958]. In each of these examples, the system (i.e., the rod) is subject to a single load: the force applied to the rod. The bifurcation behavior of a system is commonly represented in a bifurcation diagram, a plane with one axis being the load and the other axis being a quantity representing the state of the system. This quantity serves as a proxy of the state of the system, and is called the state variable, or state for brevity. The state variable can be the maximum deflection for a buckling rod, and be the total elongation for the necking rod. In the bifurcation diagram, a state of equilibrium of the system under a fixed load corresponds to a point on a curve. The bifurcation diagram of a system may contain a set of such curves. A line of constant load may intersect the curves at no point, one point, or multiple points. Thus, we usually plot the bifurcation diagrams on the force-deflection plane for a buckling rod, and on the forceelongation plane for a necking rod. The bifurcation diagram is topologically stable in that, at any value of the load, the number of states of equilibrium is independent of the choice of the quantity that represents the state of equilibrium, except for some peculiar choices. Such a bifurcation diagram works well for any system of any degrees of freedom, so long as the system is subject to a single load.

For a system subject to two independent loads, the state of equilibrium varies with both loads. The two-dimensional bifurcation diagram is inadequate. Rather, the bifurcation behavior of a two-load system is represented in a three-dimensional space, with the two loads forming the horizontal plane, and a state variable forming the vertical axis. In this three-dimensional space, 
a state of equilibrium of the system under fixed loads corresponds to a point on a surface, called the behavior surface. A given system may have several such surfaces. A line of constant loads may intersect with the behavior surfaces at no point, one point, or multiple points. The surfaces are topologically stable in that the number of states of equilibrium is independent of the choice of the quantity to represent the state. The bifurcation behavior of two-load systems has been thoroughly studied in the singularity theory, also known as the catastrophe theory [Arnol'd, 1992]. Specifically, the singularity theory proves that the projection of the behavior surfaces to the load plane results in singularity of two types: fold and cusp [Zeeman, 1979]. This projection may be used to design loading paths to avoid or harness the bifurcation.

A transducer is a system that couples two loads. An electromechanical transducer, for example, couples a mechanical force and an electrical voltage. In particular, a dielectric elastomer deforms under both mechanical force and electrical voltage. Attributes of a dielectric elastomer transducer include large deformation, high energy density, low cost, and fast response [Pelrine et al., 2000; Brochu and Pei, 2010; O’Halloran, 2008]. Applications include actuators [Anderson et al., 2012; Giousouf and Kovacs, 2013], generators [Chiba et al., 2011; Kaltseis et al., 2014], and sensors [Mannsfeld et al., 2010; Viry L. et al., 2014]. In such a transducer, a membrane of dielectric elastomer is sandwiched between two compliant electrodes. An applied voltage decreases the membrane thickness and expands the membrane in area. Such a dielectric elastomer transducer is a nonlinear, two-load system, and can exhibit rich varieties of bifurcation behavior [Goulbourne et al., 2005; Suo, 2010; Zhao and Wang, 2014]. When the membrane is subject to an equal biaxial mechanical force, the force-stretch curve is monotonic. When the membrane is subject to a voltage, the voltage-stretch curve is non-monotonic [Zhao and Suo, 2010; Plante and Dubowsky, 2006]. When the membrane is subject to both a force and a voltage, the response will change from being monotonic to being non-monotonic [Koh et al., 2011; Lu et al., 2012]. 
Here we identify the fold and cusp for a dielectric elastomer transducer by a combination of theory and experiment. For a dielectric elastomer membrane under a mechanical force $P$ and an electrical voltage $\Phi$, we choose the stretch $\lambda$ as the proxy of the state of the system. We represent the bifurcation behavior by a surface in the three-dimensional space, with $P$, $\Phi$ and $\lambda$ being the axes. We calculate the surface using the theory of ideal dielectric elastomer, and then project the surface to the load plane $(P, \Phi)$. Our experimental data agree well with the prediction of the singularity theory.

\section{Theoretical analysis}

This paper studies electromechanical catastrophe using a model system: a circular membrane of dielectric elastomer, subject to a force $P$ and a voltage $\Phi$, undergoing homogeneous and equal-biaxial deformation (Fig. 1). In the reference state, the membrane is undeformed, with radius $R$ and thickness $H$. In the deformed state, the radius becomes $r$ and the thickness becomes $h$. The elastomer is incompressible, so that $r^{2} h=R^{2} H$. The deformation is homogeneous and equal-biaxial, so that the in-plane stretches are given by $\lambda_{1}=\lambda_{2}=\lambda=r / R=(H / h)^{1 / 2}$. The mechanical force $P$ is applied through strings attached to

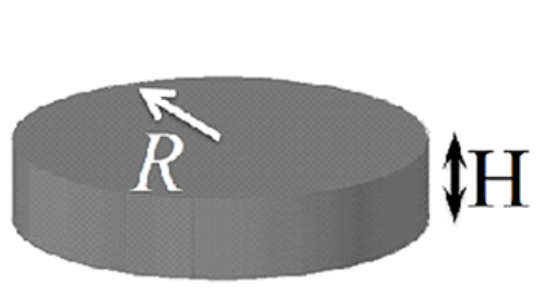

Reference state

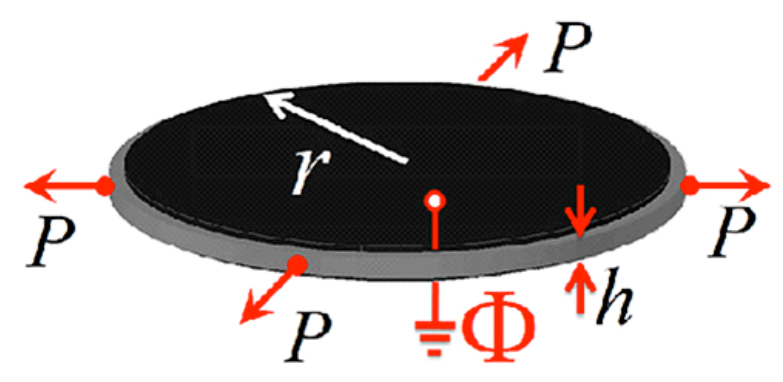

Actuated state

Fig. 1 A membrane of dielectric elastomer is subject to two loads: an equal-biaxial mechanal force applied in the plane, and an electrical voltage applied across the thickness. 
the perimeter of the circular membrane, giving the membrane a state of equal-biaxial true stress: $\sigma_{1}=\sigma_{2}=\sigma=P / 2 \pi r h=P \lambda^{2} / 2 \pi r H$. The electrical voltage $\Phi$ is applied through the thickness of the membrane, giving the membrane a state of true electric field: $E=\Phi / h=\lambda^{2} \Phi / H$

We adopt the theory of ideal dielectric elastomer [Zhao et al., 2007]. This theory assumes that the dielectric behavior of the elastomer is liquid like-that is, the true electric field is linearly proportional to the true electrical displacement, and the permittivity $\varepsilon$ is a constant independent of deformation. Under these assumptions, the equations of state are [Suo, 2010]

$$
\begin{aligned}
& \sigma_{1}+\varepsilon E^{2}=\lambda_{1} \frac{\partial W_{s}\left(\lambda_{1}, \lambda_{2}\right)}{\partial \lambda_{1}} \\
& \sigma_{2}+\varepsilon E^{2}=\lambda_{2} \frac{\partial W_{s}\left(\lambda_{1}, \lambda_{2}\right)}{\partial \lambda_{2}}
\end{aligned}
$$

where $W_{s}\left(\lambda_{1}, \lambda_{2}\right)$ is the density of the Helmholtz free energy associated with the elasticity of the elastomer. Equations (1) and (2) are valid for a membrane of an isotropic and incompressible dielectric elastomer, subject to mechanical stresses in the plane and electric field through the thickness. For more general models of dielectric elastomers, see the review by Suo [Suo, 2010]. The elastomer is a three-dimensional network of flexible polymer chains. The elasticity of the elastomer is entropic. Under no external force, each polymer chain coils. Under an external force, the polymer chain uncoils, and the distance between the two ends of the chain increases. As the polymer chain straightens, the end-to-end distance approaches the contour length of the chain, and the elastomer stiffens steeply. This stretch-stiffening effect markedly affects the electromechanical instability of dielectric elastomer [Zhao and Suo, 2010]. To represent this stretch-stiffening effect, here we adopt the Gent model [Gent, 1996]:

$$
W_{s}\left(\lambda_{1}, \lambda_{2}\right)=-\frac{\mu}{2} J_{\lim } \log \left(1-\frac{\lambda_{1}^{2}+\lambda_{2}^{2}+\lambda_{1}^{-2} \lambda_{2}^{-2}-3}{J_{\lim }}\right)
$$


where $\mu$ is the shear modulus and $J_{\lim }$ is a constant related to the limit of extension.

Inserting (3) into (1) and (2) we obtain that

$$
\frac{\lambda P}{2 \pi R H}+\varepsilon\left(\lambda^{2} \frac{\Phi}{H}\right)^{2}=\frac{\mu\left(\lambda^{2}-\lambda^{-4}\right)}{1-\left(2 \lambda^{2}+\lambda^{-4}-3\right) / J_{\lim }}
$$

This equation relates the three variables: the stretch $\lambda$, the mechanical force $P$, and the electrical voltage $\Phi$. We can fix one variable and plot the relation between the other two.

We first fix the force $P$ and plot the voltage-stretch relation (Fig. 2(a)). A fixed force $P$ sets a value of the prestretch, which for each curve is read from the intersection of the curve and the horizontal axis. The voltage-stretch curves start from this prestretched state as ground state. This loading condition can be found in some designs of actuators [Anderson et al., 2012; Giousouf and Kovacs, 2013]. When the force $P$ is fixed at a small value, the voltage-stretch curve is $\mathrm{N}$-shaped, going up, down, and up again. This behavior has been reported before [Zhao et al., 2007; Zhao and Suo, 2010]. The peak of the curve is a point of bifurcation. For fixed values of the force $P$ and voltage $\Phi$, three states of equilibrium exist when the voltage is slightly below the peak, but only one state of equilibrium exists when the voltage is above the peak. As the voltage increases continuously, upon crossing the peak, the stretch will snap horizontally, and stabilize in a state where stiffening effect prevails. Accompanied by the snap-through instability, the membrane will greatly decreases thickness and expands area, and often fails by electrical breakdown. When the fixed force is large, the voltage-stretch curve becomes monotonic. In designing an actuator to improve the maximum actuation strain, one may choose to avoid or harness this electromechanical instability [Huang et al., 2012; Keplinger et al., 2012; Wang et al., 2016]. 


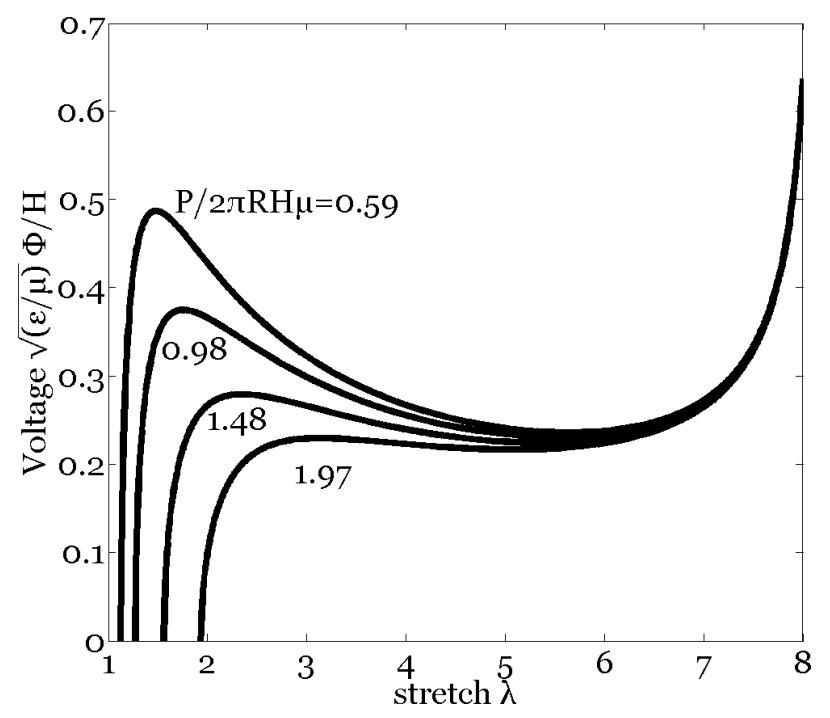

(a)

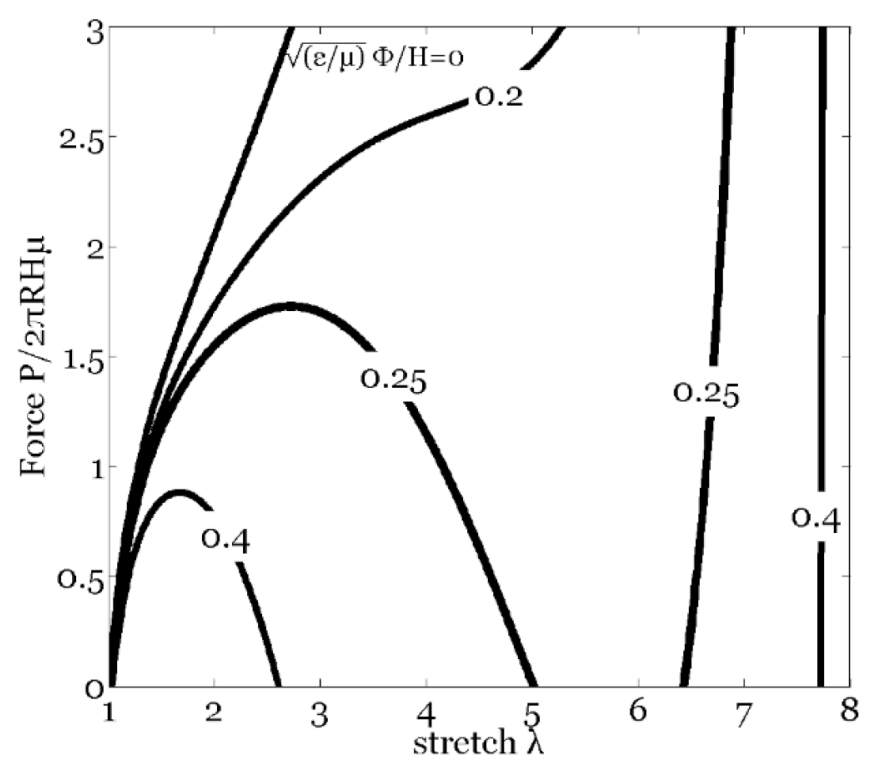

(b)

Fig. 2 States of equilibrium for a dielectric elastomer transducer. (a) Voltage-stretch curves when the membrane is under several values of constant force. (b) Force-stretch curves when the membrane is under several values of constant voltage. (c) Voltage-force curves when the membrane is under several values of constant stretch. $J_{\lim }=120$. 


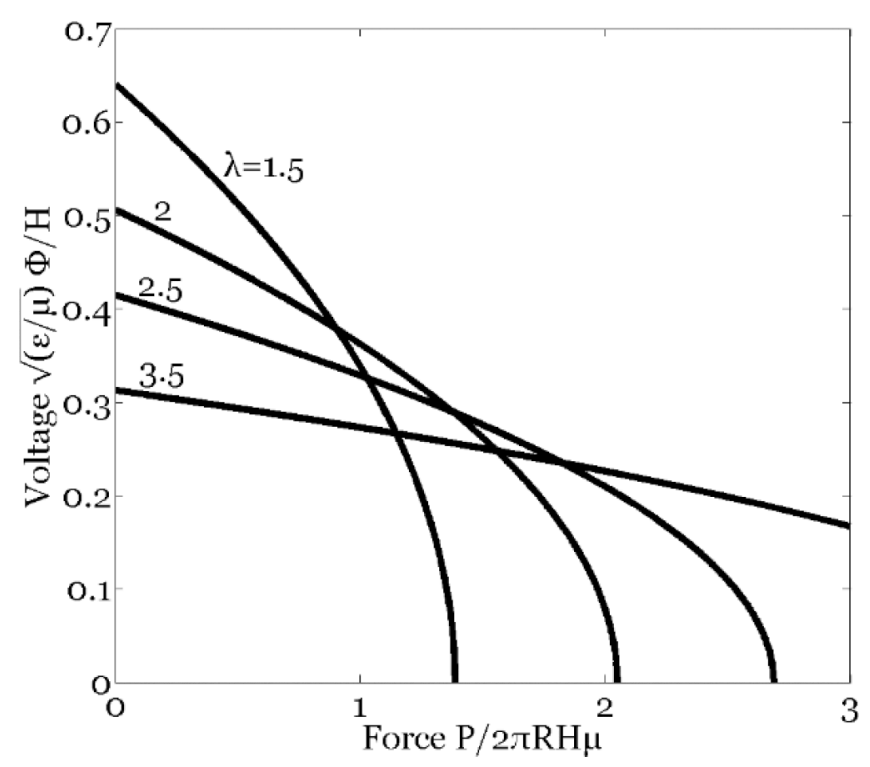

(c)

Fig. 2. (Continued)

Next we fix the electrical voltage and plot the force-stretch relation (Fig. 2(b)). A fixed voltage $\Phi$ also sets a value of the prestretch, but all the initial prestretches are close to 1 , too small to be indentified in Fig. 2(b). For example, at the normalized voltage 0.4, the prestretch is about 1.03. The force-stretch curves start from the prestretched state as ground state. This loading condition can be found in a cycle of a dielectric elastomer generator [Koh et al., 2009; Kaltseis et al., 2011; Huang et al., 2013; Lu and Suo, 2012]. When the fixed voltage is low, the force-stretch curve is monotonic. In particular, when the voltage is zero, the force-stretch curve recovers that obtained in a pure mechanical tensile testing. As the fixed voltage increases, the force-stretch curve becomes discontinuous but globally $N$-shaped. At a high fixed voltage, as the applied force increases to the peak, the stretch undergoes snap-through instability.

In the third case, we fix the stretch and plot the force-voltage relation (Fig. 2(c)). As voltage increases, the force monotonically decreases. This conclusion is readily seen in equation (4). The changes in the force and the stiffness of the membrane can be used in active vibration control and sound absorption [Heydt et al., 2000; Mckay et al., 2010]. 
Equation (4) relates the proxy of state $\lambda$ to the two loads $P$ and $\Phi$. We plot this equation as the behavior surface in a three-dimensional space, with $\lambda$ being the vertical axis (the state of the system), and with the normalized $P$ and $\Phi$ being the axes of the horizontal plane (the load plane). The behavior surface is smooth, but its projection on the load plane has the generic singularities of two types: two folds and a cusp (Fig. 3(a)). A point on the surface represents a state of equilibrium state at a pair of fixed loads. A pair of fixed force and fixed voltage corresponds to a point in the load plane, and a vertical line in the three-dimensional space. The vertical line may intersect the behavior surface at one point or three points. As the force and the voltage change, the vertical line moves. The number of states of equilibrium changes when the vertical line crosses the two folds. Each point in the $(P, \Phi)$ plane represents a pair of fixed loads. For a pair of fixed loads falling between the two folds, three states of equilibrium exist. For a pair of fixed loads falling outside the region bounded by the two folds, only one state of equilibrium exists. Equation (4) indicates that, in terms of normalized force and voltage, the folds and the cusp depend on only one material parameter, $J_{\lim }$. We plot the folds and cusp on the load plane for several representative values of $J_{\lim }$ (Fig. 3(b)).

As the two loads change continuously, a continuous sequence of states of equilibrium corresponds to a curve on the behavior surface. However, when the loads reach a fold, the system will snap. The right fold represents the snap-through conditions when we load the membrane, either mechanically or electrically, from a low level of loads to a high level. The left fold represents the snap-through conditions when we unload the membrane from a high level of loads to a low level.

Folds and cusp determine the conditions for bifurcation, and are essential to design loading paths to avoid or harness the snap-through instability. For other two-load systems, we can similarly plot the corresponding behavior surfaces, and determine the fold and cusp from the projection on the load plane. For example, for an inflated spherical dielectric balloon 
connected to a certain size of chamber, by independently controlling the pressure and the voltage, the bifurcation path can also be designed [Keplinger et al., 2012; Liang and Cai, 2015; Xie et al., 2016]. Any additional parameter, such as the size of the chamber, may strongly influence the folds and cusp [Li et al., 2013; An et al., 2015; Lu et al., 2015] .

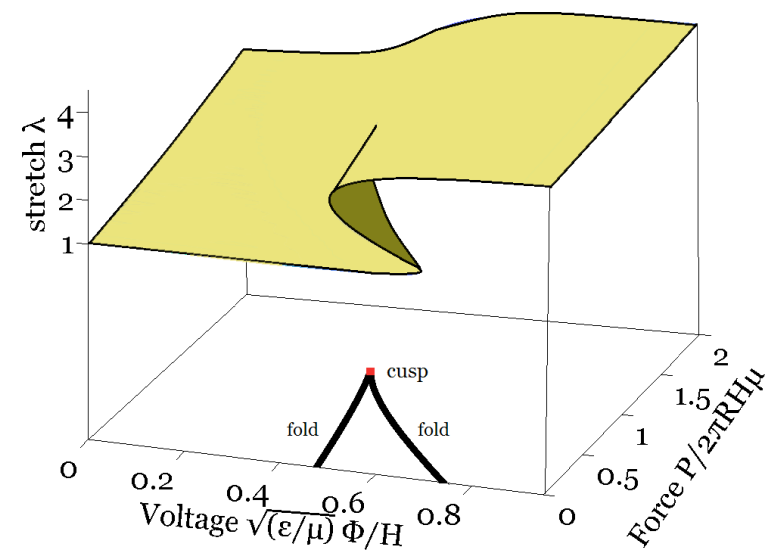

(a)

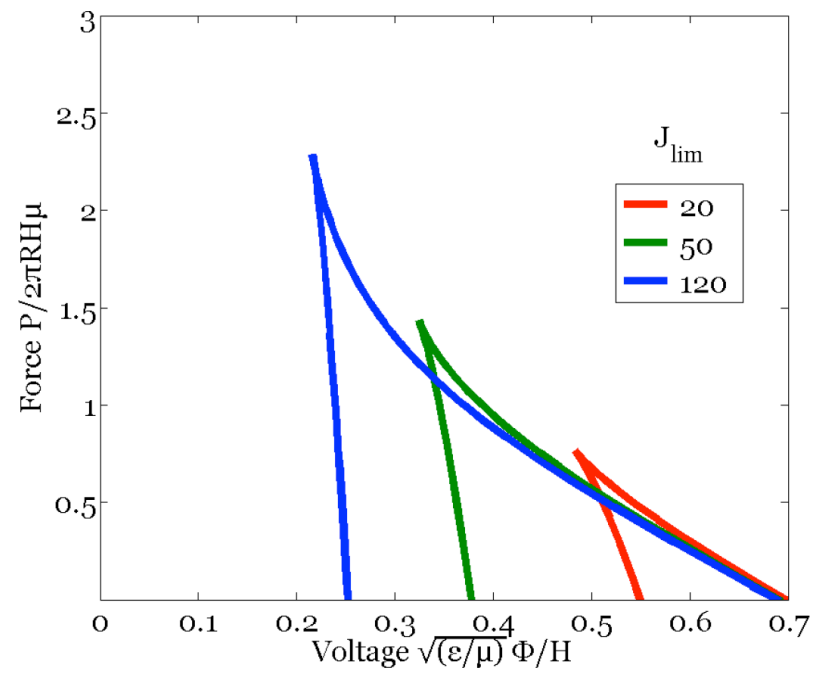

(b)

Fig. 3 Electromechanical catastrophe. (a) The behavior surface in three dimesnions for a dielectric elastomer transducer subject to a force and a voltage. The surface is smooth, but its projection on the load plane has two types of singularities: fold and cusp. $J_{\text {lim }}=120$. (b) The folds and cusp depend on the material parameter $J_{\lim }$. 


\section{Experiment and Discussion}

A circular membrane of VHB4905 (3M company) with original radius $R=15 \mathrm{~mm}$ and thickness $h=0.5 \mathrm{~mm}$ is prepared. We use a mechanism described in [Huang et al., 2012] to apply the force to the membrane. Eighteen weights uniformly connect to the boundary of the membrane through clips (Fig. 4(a)). Carbon crease of about 0.05mm thickness is brushed onto the two sides of the VHB membrane as compliant electrodes. A function generator (RIGOL DG4000) and a high voltage amplifier (TREK 30/20) are used to apply voltage across the thickness of the membrane. The voltage is linearly increased at the rate of $10 \mathrm{~V} / \mathrm{s}$. The in-plane

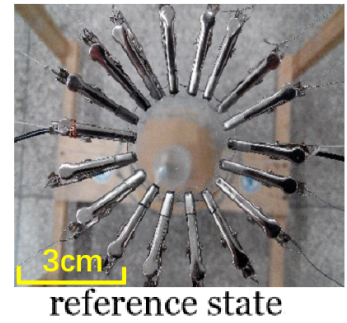

reference state

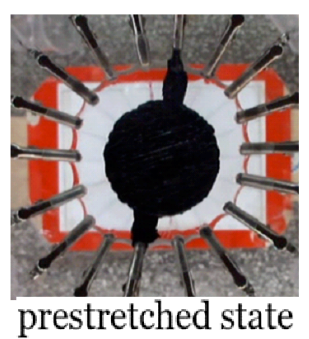

(a)

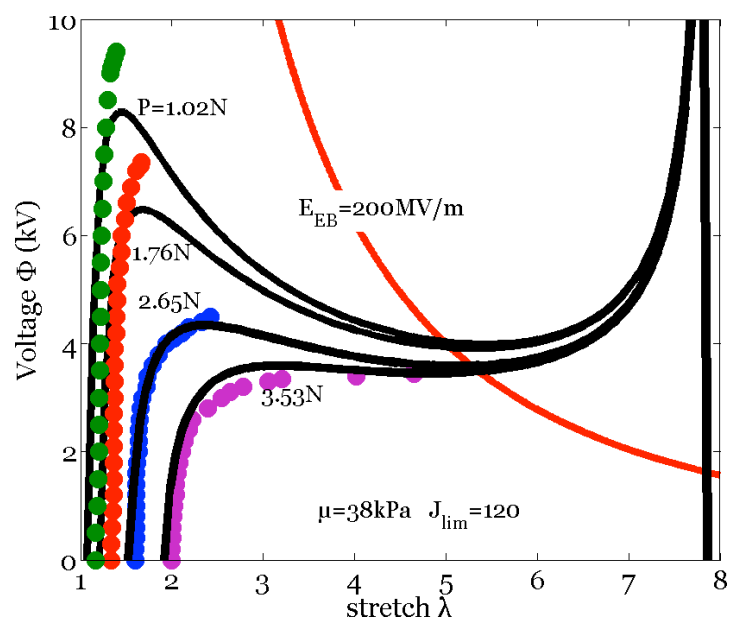

(b)

Fig. 4 (a) Photos of three states: reference state, pre-stretched state, and actuated state. The membrane is under a constant equal-biaxial force, $P=3.53 \mathrm{~N}$. (b) Voltage-stretch curves when the membrane is under different values of constant force. Solid curves are theoretical predictions, and the dots are experimental data. Also included is the curve representing the condition of electrical breakdown field. 
expansion of the membrane is recorded by a video camera. We conduct two kinds of experiments: electrical actuation under a constant force, and mechanical pulling under a constant voltage.

In electrical actuation, we fix the force at four levels and record the voltage-stretch curves until electrical breakdown occurs. Under small values of constant forces, $P=1.02 N, 1.76 N$, the membrane can sustain high voltage before electrical breakdown, but the actuation strain is small (Fig. 4(b)). Under the force $P=2.65 N$, the voltage-induced expansion of the membrane is significant. Soon after the expansion the membrane fails by electrical breakdown. Under the force $P=3 \cdot 53 N$, the membrane undergoes large deformation without snap-through instability, with radial stretch changing from $\lambda_{\text {pre }}=2.4$ to $\lambda=4 \cdot 7$. Localized wrinkles are observed before the electrical breakdown. Fig. 4(b) shows good agreement between the theoretical predictions and the experiment. In plotting equation (4), we have used the following parameters: the permittivity of VHB4905 is $\varepsilon=3 \cdot 98 \times 10^{-11} \mathrm{~F} / \mathrm{m}$, the electrical breakdown field is $E_{E B}=200 M V / m$, and the limiting stretch is $J_{\lim }=120$ [Lu et al., 2012]. In fitting the experimental data the shear modulus is taken as $\mu=38 \mathrm{kPa}$.

In mechanical pulling, we fix the voltage under four levels and record the forcedeformation curve until failure. A special case $\Phi=$ o corresponds to the purely mechanical equal biaxial tensile test. When the applied voltage is low, for example $\Phi=1000 \mathrm{~V}$, the forcedeformation curve almost coincides with the purely mechanical case (not shown in Fig. 5). Under the voltage $\Phi=3200 \mathrm{~V}$, the membrane expands as the force increases and undergoes a fast expansion when the force attains around $4 \mathrm{~N}$. Under the voltage $\Phi=3900 \mathrm{~V}$, the membrane ruptures at small deformation by electrical breakdown. Fig. 5 shows the comparison between the theoretical predictions and the experiment, where the shear modulus is taken as 
$\mu=36 \mathrm{kPa}$. The slight difference in the magnitude of shear modulus between the electrical actuation test and the mechanical pulling test is possibly due to the strong viscosity of the VHB membrane [Zhao et al., 2011; Foo and Zhang, 2015]. The viscous effects will affect the fitting shear modulus as well as the instabilities, but in this work we have lumped the viscous effects into a calibrated elastic parameter for simplicity.

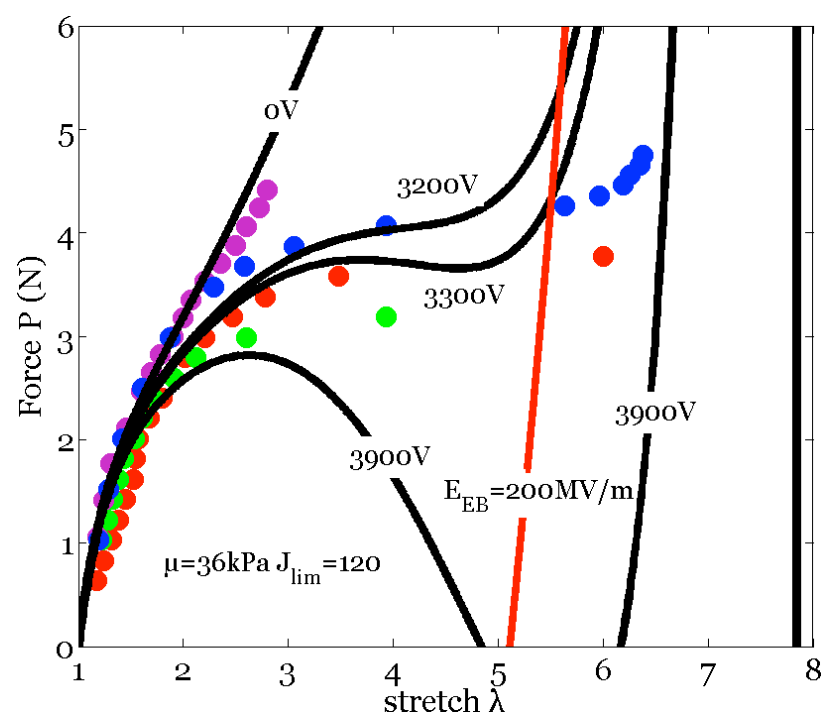

Fig. 5 Force-stretch curves when a membarne is under different values of constant voltage. Solid cuvres are theoretical predictions, and the dots are experimental data.

Comparing the electrical actuation test and the mechanical pulling test, we can find many common features. Under low levels of fixed loads (either mechanical or electrical), the stimuli-deformation curves are monotonic. Under high levels of fixed load, the electromechanical snap-through is observed: either a safe sudden snap with large deformation or an unsafe snap with failure by electrical breakdown. The theory predicts that the electromechanical folds and cusp determine the critical bifurcation conditions. We plot the experimental data of the critical conditions at failure on the load plane (Fig. 6). The black circular dots are from the electrical actuation test and the red triangle dots are from the 
mechanical pulling test. All the experimental data lie close to the right fold, validating the theoretical prediction.

We can design a loading path in the load plane to pass over the cusp to avoid snapthrough instability. For example, when we first apply a force $P=3.53 \mathrm{~N}$ and hold the force at the fixed value, and then apply the voltage, we can achieve a large stretch of actuation (Fig. 4(b)). In hindsight, we may say that overpassing the cusp is responsible for the initial discovery of the prestretch effect on large actuation made by Pelrine et al [Pelrine et al., 2000].

In our experiment we have also attempted another loading path as follows. Apply a mechanical force $P$ to a value above the cusp, fix the force and increase the voltage $\Phi$ to overpass the cusp, and then fix the voltage and decrease the force to zero. In the final state, by increasing and then decreasing the force back to zero, we hoped to achieve a large deformation only under a voltage. However, limited to the low electrical breakdown strength of the VHB membrane, we did not succeed in observing this large deformation following this special loading path in our experiment. For the same reason, we were unsuccessful to probe the left fold in our experiment.

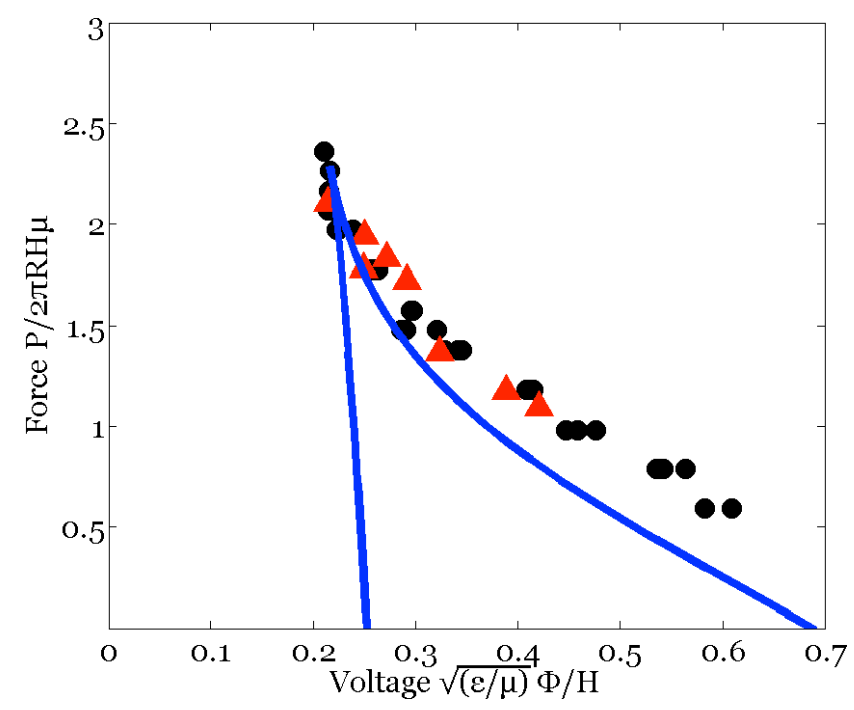

Fig. 6 Folds and cusp on the load plane. The solid curves are theoretical predictions. Black dots are the recorded maximum voltage before failure when a membrane is under a constant force. Red triangles are the recorded maximum force before failure when a membrane is under a constant voltage. 


\section{Conclusion}

The electromechanical catastrophe theory can be applied to a system of arbitrary shape, so long as the system is subject to two independent loads. For a system of a few degrees of freedom, the states of equilibrium may be obtained analytically. For a system of many degrees of freedom, the states of equilibrium must be obtained by numerical analyses. As described in Introduction, the singularity theory shows that the bifurcation behavior for any two-load system, however complex, contains two types of singularity: fold and cusp. This paper uses a simple system to illustrate this behavior.

We study a membrane of dielectric elastomer subject to an equal biaxial force and a voltage across the thickness. For such a two-load system, we construct a three-dimensional space, with one axis being the stretch, and the other two axes being the electrical and mechanical loads. In the three-dimensional space, a state of equilibrium at fixed loads corresponds to a point on a surface. We project the surface onto the voltage-stretch plane, forcestretch plane, and the force-voltage plane. We find that the behaviors of voltage-induced deformation under a constant force and the force-induced deformation under a constant voltage are similar: under low levels of given loads, either mechanical or electrical, the stimulideformation behavior is monotonic and stable. Under high levels of given loads, the behavior becomes non-monotonic and unstable. The projection of the state surface on the load plane determines the critical conditions of bifurcation as two folds and one cusp, which can be used to design loading paths to avoid or harness the electromechanical bifurcation. We conduct two kinds of experiments: electrical actuation under a constant force and mechanical pulling under a constant voltage. Good agreement between the theory and the experiment is obtained both on the deformation history and the determination of folds and cusp.

\section{Acknowledgments}


The work was supported by the National Natural Science Foundation of China (No. 11402185 and No. 11321062). TQ acknowledges the support by the open fund of Key Laboratory

of Soft Machines and Smart Devices of Zhejiang Province. ZS acknowledges the support of MRSEC (DMR 14-20570) and a visiting appointment to International Center for Applied Mechanics at Xian Jiaotong University.

\section{References}

An, L., Wang, F., Cheng, S., Lu, T., \& Wang, T. J., 2015. Experimental investigation of the electromechanical phase transition in a dielectric elastomer tube. Smart Materials and Structures, 24(3), 035006.

Anderson, I. A., Gisby, T. A., McKay, T. G., O’Brien, B. M., Calius, E. P., 2012. Multi-functional dielectric elastomer artificial muscles for soft and smart machines. Journal of Applied Physics, 112(4), 041101.

Arnol'd, V. I., 2003. Catastrophe theory. Springer Science \& Business Media.

Brochu, P., Pei, Q., 2010. Advances in dielectric elastomers for actuators and artificial muscles. Macromolecular rapid communications, 31(1), 10-36.

Chiba, S., Waki, M., Kornbluh, R., Pelrine, R., 2011. Current status and future prospects of power generators using dielectric elastomers. Smart Materials and Structures, 20(12), 124006.

Foo, C. C., Zhang Z. Q., 2015. A finite element method for inhomogeneous deformation of viscoelastic dielectric elastomers. International Journal of Applied Mechanics, 7(5).

Gent, A. N., 1996. A new constitutive relation for rubber. Rubber chemistry and technology, 69(1), 59-61.

Giousouf, M., Kovacs, G., 2013. Dielectric elastomer actuators used for pneumatic valve technology. Smart Materials and Structures, 22(10), 104010.

Goulbourne, N., Mockensturm, E., \& Frecker, M., 2005. A nonlinear model for dielectric elastomer membranes. Journal of Applied Mechanics, 72(6), 899-906.

Heydt, R., Pelrine, R., Joseph, J., Eckerle, J., Kornbluh, R., 2000. Acoustical performance of an electrostrictive polymer film loudspeaker. The Journal of the Acoustical Society of America, $107(2), 833-839$.

Hill, R., 1958. A general theory of uniqueness and stability in elastic-plastic solids. Journal of the Mechanics and Physics of Solids, 6(3), 236-249. 
Huang, J., Li, T., Foo, C. C., Zhu, J., Clarke, D. R., Suo, Z., 2012. Giant, voltage-actuated deformation of a dielectric elastomer under dead load. Applied Physics Letters, 100(4), 041911.

Huang, J., Shian, S., Suo, Z., Clarke, D. R., 2013. Maximizing the Energy Density of Dielectric Elastomer Generators Using Equi - Biaxial Loading. Advanced Functional Materials, 23(40), 5056-5061.

Kaltseis, R., Keplinger, C., Baumgartner, R., Kaltenbrunner, M., Li, T., Mächler, P., Schwodiauer, R., Suo, Z. G., Bauer, S., 2011. Method for measuring energy generation and efficiency of dielectric elastomer generators. Applied Physics Letters, 99(16), 162904.

Kaltseis, R., Keplinger, C., Koh, S. J. A., Baumgartner, R., Goh, Y. F., Ng, W. H., Kogler, A., Tröls, A., Foo, C. C., Suo, Z. G., Bauer, S., 2014. Natural rubber for sustainable high-power electrical energy generation. RSC Advances, 4(53), 27905-27913.

Keplinger, C., Li, T., Baumgartner, R., Suo, Z., \& Bauer, S., 2012. Harnessing snap-through instability in soft dielectrics to achieve giant voltage-triggered deformation. Soft Matter, 8(2), 285-288.

Koh, S. J. A., Li, T., Zhou, J., Zhao, X., Hong, W., Zhu, J., Suo, Z., 2011. Mechanisms of large actuation strain in dielectric elastomers. Journal of Polymer Science Part B: Polymer Physics, 49(7), 504-515.

Koh, S. J. A., Zhao, X., Suo, Z., 2009. Maximal energy that can be converted by a dielectric elastomer generator. Applied Physics Letters, 94(26), 262902.

Landau, L. D., Lifshitz, E. M., 1986. Theory of elasticity.

Li, T., Keplinger, C., Baumgartner, R., Bauer, S., Yang, W., Suo, Z., 2013. Giant voltage-induced deformation in dielectric elastomers near the verge of snap-through instability. Journal of the Mechanics and Physics of Solids, 61(2), 611-628.

Liang, X., Cai, S. 2015. Shape bifurcation of a spherical dielectric elastomer balloon under the actions of internal pressure and electric voltage. Journal of Applied Mechanics, 82(10), 101002.

Lu, T., Suo, Z., 2012. Large conversion of energy in dielectric elastomers by electromechanical phase transition. Acta Mechanica Sinica, 28(4), 1106-1114.

Lu, T., Huang, J., Jordi, C., Kovacs, G., Huang, R., Clarke, D. R., Suo, Z., 2012. Dielectric elastomer actuators under equal-biaxial forces, uniaxial forces, and uniaxial constraint of stiff fibers. Soft Matter, 8(22), 6167-6173. 
Lu, T., An, L., Li, J., Yuan, C., Wang T. J., 2015. Electro-mechanical coupling bifurcation and bulging propagation in a cylindrical dielectric elastomer tube. Journal of the Mechanics and Physics of Solids, 85, 160-175.

Mannsfeld, S. C., Tee, B. C., Stoltenberg, R. M., Chen, C. V. H., Barman, S., Muir, B. V., Anatoliy, N. S., Colin, R., Bao, Z., 2010. Highly sensitive flexible pressure sensors with microstructured rubber dielectric layers. Nature materials, 9(10), 859-864.

McKay, T., O’Brien, B., Calius, E., \& Anderson, I., 2010. An integrated, self-priming dielectric elastomer generator. Applied Physics Letters, 97(6), 062911.

O'Halloran, A., O’malley, F., McHugh, P., 2008. A review on dielectric elastomer actuators, technology, applications, and challenges. Journal of Applied Physics, 104(7), 071101.

Pelrine, R., Kornbluh, R., Pei, Q., Joseph, J., 2000. High-speed electrically actuated elastomers with strain greater than 100\%. Science, 287(5454), 836-839.

Plante, J. S., Dubowsky, S., 2006. Large-scale failure modes of dielectric elastomer actuators. International Journal of Solids and Structures, 43(25), 7727-7751.

Suo, Z., 2010. Theory of dielectric elastomers. Acta Mechanica Solida Sinica, 23(6), 549-578.

Viry, L., Levi, A., Totaro, M., Mondini, A., Mattoli, V., Mazzolai, B., Beccai, L., 2014. Flexible three - axial force sensor for soft and highly sensitive artificial touch. Advanced Materials, 26(17), 2659-2664.

Wang, F. F., Lu, T., Wang, T. J., 2016. Nonlinear vibration of dielectric elastomer incorporating strain stiffening. International Journal of Solids and Structures , 87, 70-80.

Xie, Y. X., Liu, J. C., Fu, Y. B., 2016. Bifurcation of a dielectric elastomer balloon under pressurized inflation and electric actuation. International Journal of Solids and Structures, $78,182-188$.

Zeeman, E. C., 1979. Catastrophe theory. In Structural Stability in Physics (pp. 12-22). Springer Berlin Heidelberg.

Zhao, X., Hong, W., Suo, Z., 2007. Electromechanical hysteresis and coexistent states in dielectric elastomers. Physical review B, 76(13), 134113.

Zhao, X., Koh, S. J. A., Suo, Z., 2011. Nonequilibrium theormodynamics of dielectric elastomers. Internatioanl Journal of Applied Mechanics, 3(2), 203-207.

Zhao, X., Suo, Z., 2010. Theory of dielectric elastomers capable of giant deformation of actuation. Physical Review Letters, 104(17), 178302.

Zhao, X., Wang, Q., 2014. Harnessing large deformation and instabilities of soft dielectrics: theory, experiment, and application. Applied Physics Reviews, 1(2), 021304. 
